\title{
Efficacy of Fine Needle Aspiration Cytology in Diagnosis of Salivary Gland Tumors
}

\author{
lacob Alina*, Sin Anca, Mezei Tibor, Mocan Simona, Ormenisan Alina, Tilinca Mariana \\ University of Medicine and Pharmacy, Tîrgu Mureș, România
}

Introduction: Masses of the head and neck comprise a variety of benign and malignant tumors and tumor-like conditions, which may pres-
ent diagnostic challenges to the surgeon and pathologist as well. Fine needle aspiration cytology is considered to be a valuable diagnostic
tool used for preoperative evaluation of various masses in the head and neck region. However, its role is quite controversial for salivary gland
tumour evaluation. This study was aimed to evaluate salivary gland fine-needle aspiration cytology for sensitivity, specificity and diagnostic
accuracy in our medical center.

Material and method: The present study included 58 patients with different salivary gland lesions who underwent preoperative fine-needle aspiration procedure followed by surgical procedure and histological examination. The cytological findings were compared with the final histological diagnosis and concordance assessed.

Results: Of the 58 cases with salivary gland lesions, most of them involved parotid gland (72.41\%), 91.38\% were neoplasms and $8.62 \%$ were non-neoplastic lesions. Out of 53 neoplasms, 39 were benign and 14 were malignant salivary gland tumors. The sensitivity and a specificity of fine-needle aspiration cytology in differentiation malignancies from benign lesions was $100 \%$ and $97.73 \%$ respectively. In some situations cytological features could not provide correct tumor characterization.

Conclusions: Fine-needle aspiration cytology is a simple and reliable technique for preoperative evaluation of salivary gland tumors with a high sensitivity diagnosis of malignant tumors, but a lower tumor type characterization. Due to the minimally invasive nature of the technique, fine-needle aspiration cytology offers valuable information for planning of subsequent therapeutic management.

Keywords: fine-needle aspiration, cytology, salivary gland tumors

Received: 09 December 2014 / Accepted: 11 August 2015

\section{Introduction}

Salivary gland tumors are relatively rare and represent approximately $3-6 \%$ of head and neck tumors $[1,2]$. The annual worldwide incidence of all salivary gland tumors varied between $0.4-13.5$ cases per 100.000 population. There is also some geographic variation in the incidence of tumour types. The frequency of malignant salivary neoplasms ranges from 0.4-2.6 cases per 100,000 population [1]. Most salivary gland masses (about $80 \%$ ) involve the parotid gland and $80 \%$ of them are benign [3].

In 2005 the World Health Organization has classified these tumors in 10 benign and 23 malignant entities all of epithelial origin. Non-epithelial tumors are very rare about $2-5 \%$ of all salivary tumor pathology. Although this classification is complex, it has advantages regarding the prognostic and therapeutic aspects, because the biological behavior of each tumor type is different $[1,4]$.

The large histological variety of benign and malignant salivary gland neoplasms and the lack of specific tumor markers are increasing the difficulty of their diagnosis. On the other hand, salivary glands are easily accessible, so they are very good targets for fine-needle aspiration. This procedure is a simple and useful diagnostic method used in preoperative evaluation of different tumor masses in the head and neck area.

* Correspondence to: Alina lacob

E-mail: czmaalina@yahoo.com
The history of fine needle aspiration cytology goes back to the interwar period where it came into use concomitant in the United States and Europe [5,6], however Zajicek from Karolinska Hospital in Stockholm brought the aspiration cytology to international attention by his publications [7].

The most important aspect in interpreting data obtained by fine-needle aspiration cytology is differentiating the benign tumors from those malignant, and then differentiating the various entities [8]. This is considered a diagnostic method based on cyto-morphological aspect of cells and tissue particles acquired using a fine needle. The incisional biopsy is no longer justified because of the risk of tumor spillage and damage to the facial nerve if it is used in parotid gland [5].

Fine-needle aspiration is a safe procedure with minimal incidence of complications such as minor local bleeding and inflammatory reaction, and practical with no risk of tumor contamination on the needle track $[5,8]$.

The effectiveness of fine-needle aspiration cytology in interpretation of the morphology of salivary gland tumors is still controversial. However, this procedure helps to differentiate between inflammatory process which may not require surgery and neoplastic lesions. On the other hand, it can distinguish benign from malignant tumors in this way allowing an adequate planning of the treatment [5].

The present study tries to assess the importance of fineneedle aspiration procedure performed preoperative in or- 
der to evaluate patients with salivary gland tumors and to identify the specific lesions in which cytological examination can be use as a diagnostic tool.

\section{Methods}

A clinical prospective study was conducted between May 2012 - February 2014 at the Department of Oral and Maxillo-Facial Surgery at the County Emergency Clinical Hospital of Târgu-Mureș, Romania.

The study included 58 patients that met the following inclusion criteria: tumors or tumor-like masses of the salivary glands that were not ulcerated, completely covered by intact skin or mucosa. The fine-needle aspiration procedure was explained to patients and a written consent was obtained from them. Approval of the University's Ethics Committee was also obtained. The study was conducted in accordance of ethical and deontological standards set by the University of Medicine and Pharmacy of Târgu-Mureș regarding clinical studies. Exclusion criteria were the following: inadequate cytology slides for interpretation, patients with recurrent lesions or who have not received surgical treatment and histological result, or those with contraindications for performing fine-needle aspiration.

In all cases, fine needle aspiration procedure was performed as a part of preoperative assessment. The procedure was done by maxillofacial surgeon without ultrasound guidance, using a $23 \mathrm{G}$ needle and $10 \mathrm{ml}$ or $20 \mathrm{ml}$ syringe unattached to an aspiration syringe holder. A minimum of two needle passes were made in each case, in order to obtain enough biological material for adequate cytological interpretation. The aspirated material was spread onto 2-4 slides and fixed immediately by immersion in ethanol 95\%. In the same day the slides were sent for staining (Papanicolaou) and interpretation at the Department of Pathology from the same hospital. Subsequently, patients sustained surgical treatment and histological specimens obtained were analyzed in the Pathology Department by a different pathologist. Wherever required, special stains and immunohistochemical analyses were done.

Table I. Cytohistological correlation in salivary gland tumors.

\begin{tabular}{lccccc}
\hline Histological diagnosis & No. of & Concordant & \multicolumn{2}{c}{ Discordant cytology } \\
\cline { 4 - 5 } & cases & cytology & Benign & Malignant \\
\hline Sialadenitis & 5 & 5 & - & - \\
Pleomorphic adenoma & 17 & 16 & 1 & - \\
Warthin tumor & 12 & 8 & 4 & - \\
Benign lymphoepithelial lesion & 3 & 3 & - & - \\
Lymphoepithelial cyst & 3 & - & 3 & - \\
Pseudocyst & 1 & - & 1 & - \\
Schwannoma & 1 & - & 1 & - \\
Dermoid cyst & 1 & - & 1 & - \\
Desmoid fibromatosis & 1 & - & 1 & - \\
Lymphoma & 3 & - & - & 3 \\
Myoepithelial carcinoma & 1 & - & - & 1 \\
Squamous cell carcinoma & 8 & 7 & - & 1 \\
Adenoid cystic carcinoma & 1 & - & 1 & - \\
Oncocytic carcinoma & 1 & 1 & - & - \\
\hline
\end{tabular}

The extension of the surgical procedure was decided upon cytological and clinical data. Cytological result obtained by fine-needle aspiration was compared in each case to the final histological result.

Statistical analysis was performed using GraphPad InStat 3 and Medcalc softwares. Analysis and evaluation of qualitative variables and statistically significant differences of groups were determined using Chi-square test. The level of significance was set at $\mathrm{p}<0.05$.

In order to assess the performance of fine-needle aspiration procedure as a diagnostic test for salivary gland tumors indicators of sensitivity (Se), specificity (Sp), positive predictive value (PPV) were calculated for $95 \%$ confidence intervals.

\section{Results}

The study included 58 patients between ages 10 and 92 years. They were admitted to be treated for different salivary gland masses. There was no gender predilection ( 27 males $-46.55 \%$ and 31 females $-53.45 \%$ ), with male to female ratio of $1: 1.4$.

Salivary gland tumors involved mainly the parotid gland in 42 cases (72.41\%), submandibular gland was interested in 14 cases $(24.14 \%)$ and minor salivary glands (palate) in 2 cases $(3.45 \%)$.

Of the 58 tumors studied, 53 were neoplastic lesions $(91.38 \%)$ benign or malignant histologically confirmed and only 5 were non-neoplastic (inflammatory) lesions (8.62\%). Of all neoplastic lesions, 39 (73.58\%) were found to be benign, and $14(26.42 \%)$ were malignant tumors.

Following the distribution by age of benign and malignant salivary gland tumors, were found only benign tumors in age under 50 , with the emergence of malignant tumors in age groups after 51 years, their incidence increasing progressively with age.

Of the 44 cases of benign salivary gland lesions ( 5 inflammatory lesions and 39 benign tumors), cytological features were concordant with histological result in 32 cases $(72.73 \%)$. For the remaining 12 cases $(27.27 \%)$ cytological smears could only describe the cellular populations which were suggestive of benign entities (Table I).

Pleomorphic adenoma was the most common in benign tumor group (17 cases), followed by Warthin's tumor (12 cases). The concordant cytological diagnosis and tumor characterization were adequate in 16 cases of pleomorphic adenoma and 8 cases of Warthin's tumor. There were three situations with non-salivary tumors located into glandular parenchyma which were difficult to be diagnosed on cytology due to lack of cyto-morphological features. In these cases of schwannoma, dermoid cyst and desmoid fibromatosis the cytology smears were suggestive for benign lesion in each situation but only the histological studies provided the correct tumor characterization.

In the studied salivary gland tumors there were 14 cases of malignancies based on histological diagnosis. In 8 cases the cytological diagnosis was concordant with histology. In 
5 situations the cytological aspect was suggestive for malignancy but no further characterization of the lesion could be done (Table I). One discordant case which presented only inflammatory features on cytology was diagnosed to be adenoid cystic carcinoma after histology. The malignant area was estimated to be very small (about $4 \mathrm{X} 2 \mathrm{~mm}$ ) surrounded by inflammation.

Statistical analysis was performed to compare the results of histological and cytological examinations. Using Chi-square test was obtained a value of $\mathrm{p}=0.0001$, highly significant statistically which means that in case of salivary gland tumors the values of cytology results were very close to those of the histology.

Cytological examination had a sensitivity of $100 \%$ and a specificity of $97.73 \%$ for diagnosis of malignant salivary gland lesions. Positive predictive value was $93.3 \%$ and negative predictive value was $100 \%$.

\section{Discussion}

Fine-needle aspiration technique is used as an important diagnostic tool in the management of various head and neck lesions $[2,5]$.

There are many authors who consider aspiration cytology as a useful preoperative step in the assessment of salivary gland lesions, but others believe that the method has limited clinical application because of the false-positive and false-negative rate. However, preoperative cytological evaluation has a great importance in diagnosis and differentiation of benign from malignant lesions [5].

Few submandibular masses are considered to be primary tumors of the salivary gland. Most of them are due to inflammatory diseases or malignancies involving the lymph nodes in the region. Fine-needle aspiration points is useful to distinguish these entities and guiding the therapy. Even if the result is negative, should not be underestimated the clinical reasoning that suspected a malignancy [2].

In the present study was found concordant cytologic diagnosis and tumor characterization in 40 cases (69\%).

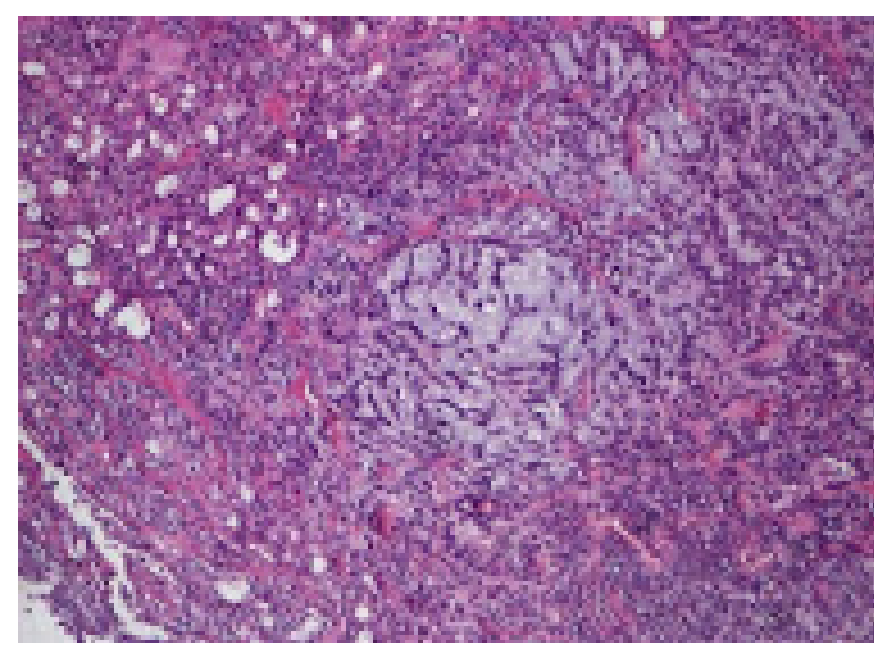

Fig. 1. Pleomorphic adenoma (benign mixed tumor), histology. A somewhat more cellular tumor is pictured with biphasic population of epithelial and mesenchymal cells. Stroma is myxoid with some hyaline areas present. (obj. 10x, HE stain)
In 17 cases $(29.3 \%)$ the cytology could only describe the cellular population without further tumor typing, but the diagnosis of benign or malignant tumor was right. In these cases the final diagnosis could be made only on histology. There was one discordant case of malignant tumor misdiagnosed as inflammation on cytology. In cases of inadequate samples or insufficient material for correct cytological interpretation, fine-needle aspiration procedure can be repeated to improve the cytological diagnosis. Brennan et al evaluated the utility of repeat fine-needle aspiration in cytological diagnosis of salivary gland lesions for unclear cases in order to improve the diagnosing accuracy. However, the authors have reported the sensitivity and specificity in differentiation malignant from benign lesions similar to the initial examination $(70 \%$ and $95 \%$ before, respectively $84 \%$ and $93 \%$ after repeated aspiration) [9].

Observing the various pathological entities in patients included in our study were found $8.62 \%$ non-neoplastic (inflammatory) lesions such as sialadenitis. The proportion is different with the existing literature. Ashraf et al have reported 14\% non-neoplastic lesions [10]. Jain et al presented a study with $10 \%$ non-neoplastic lesions [11], and Singh et al published a study with 55.9\% inflammatory lesions [12].

In the studied group were found $91.38 \%$ neoplastic lesions - benign and malignant tumors. Of these cases, $73.58 \%$ were benign and $26.42 \%$ were found to be malignant tumors. Most authors reported quite similar data: $79-89.5 \%$ benign and $10.5-21 \%$ malignant masses $[10,11,13,14]$.

In our study, benign tumor entity with the highest frequency was pleomorphic adenoma (40\%), followed by Warthin's tumor. Cyto-histologic correlation for pleomorphic adenoma diagnosis was obtained in $91.6 \%$ of cases. This is similar to previous reports $[10,11]$. Pleomorphic adenoma presents an interesting cyto-morphological diversity consisting in a mixture of myoepithelial and ductal cells in varying proportions, placed in a fibroconjunctive

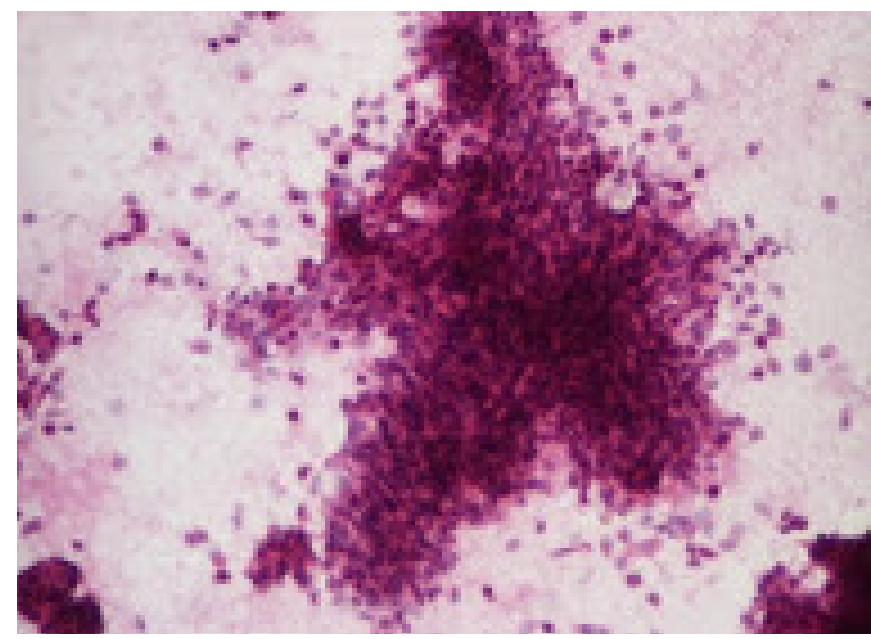

Fig. 2. Pleomorphic adenoma, cytology. Bland epithelial cells together with mesenchymal cells. Stroma fragments are not observed in this image (obj. 20x, Papanicolaou stain) 


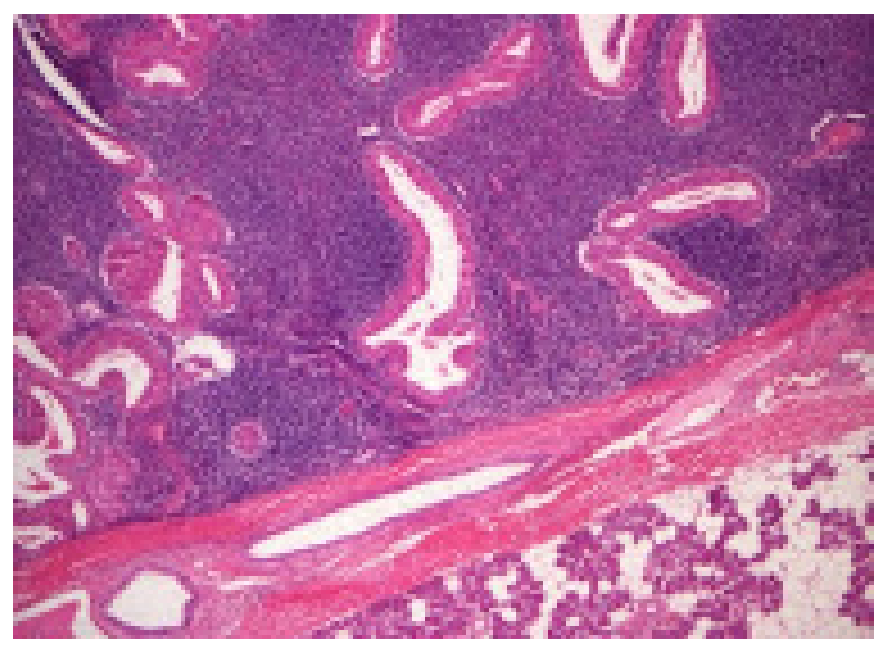

Fig. 3. Warthin-tumor, histology. Partial cystic spaces lined by double layer of epithelial cells resting on dense lymphoid stroma with variable germinal centers with occasional polypoid projections. Epithelial cells present surface palisading and have an oncocytic columnar cell appearance with underlying discontinuous basal cells (obj. 10x, HE stain)

matrix, with myxoid, chondroid or mucoid stroma (Figure 1 and Figure 2). Myoepithelial cells might be plasmacytoid or spindled giving rise to a variety of incorrect interpretations $[15,16]$.

Warthin tumor is histological characterized by cystic spaces surrounded by a double layer of oncocytic epithelial cells which can develop papillary projections into the cavities (Figure 3). A variable amount of lymphoid tissue is present. However, the absence of the epithelium on cytological smears may be interpreted as an inflammatory process [17] (Figure 4).

Of salivary gland malignant tumors, specific elements of squamous cell carcinoma were histological diagnosed in 8 cases $(15.7 \%)$, differentiation of primary tumors from those metastatic being difficult only by cyto-morphological analysis [18].

Fine-needle aspiration usefulness in diagnosis of lymphoma is limited without ancillary techniques (flow-cytometry) $[19,20]$. Cyto-morphological features of these particular entities do not provide sufficient evidence for a rigorous diagnosis and classification. Because of particular histology of lymphomas and the complexity of their classification the final diagnosis of these cases remains histological [21]. However, fine-needle aspiration cytology can be use for the initial assessment and management of these patients [22-24].

Various clinical trials reported a rate of false-negative cytological diagnosis of between $0 \%$ and $37 \%$ [10,11]. The present study had $7.1 \%$ rate of false-negative cytological diagnosis due to a case of adenoid carcinoma which has been interpreted as inflammatory lesion.

The sensitivity of fine-needle aspiration cytology in differentiation of malignant from benign lesions in studied group was $100 \%$. The specificity in the same situation was $97.73 \%$. The positive predictive value was $93.3 \%$ and the

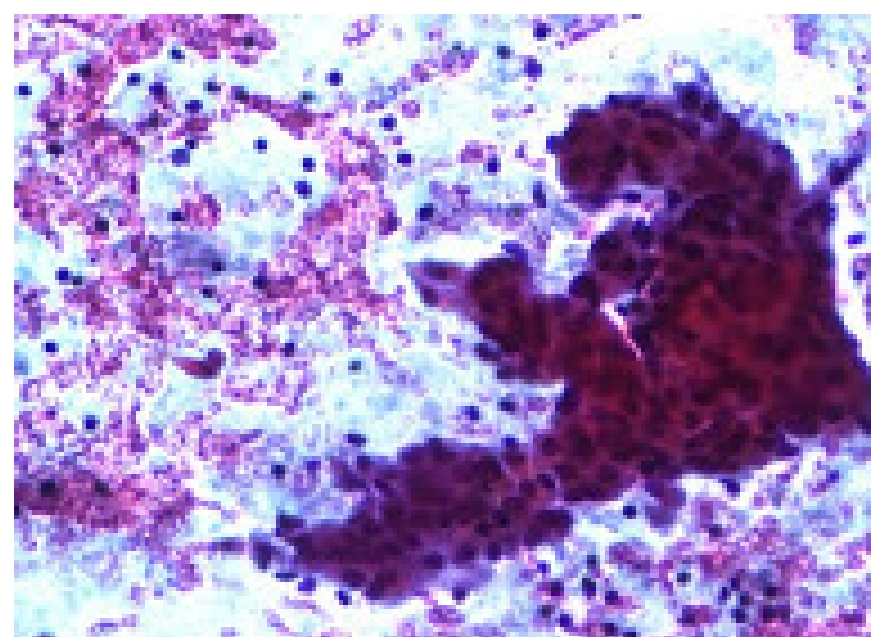

Fig. 4. Warthin-tumor, cytology. Oncocytic cell groups in a lymphoid background dominated by small mature lymphocytes (obj. 20x, Papanicolaou stain)

negative predictive value was $100 \%$. Numerous clinical trials have reported the accuracy of cytological diagnosis of salivary gland neoplasms as being $86-98 \%$. The sensitivity has ranged from $62-97.6 \%$ and specificity from $94.3 \%$ to $100 \%[2,10,11,14,25]$.

The only relative contraindication of the performing the fine-needle aspiration procedure is the hemorrhagic disease. In our series no complications such as tumor cells seading, hematoma, nerve damage, or infection were observed [26].

The role of fine-needle aspiration in the diagnosis and management of salivary glands tumors is not well established. The usual recommendation for salivary glands tumors is surgical excision. However, cytological diagnosis of benign lesion in poor risk patients could be of benefit in avoiding inappropriate surgery [5].

\section{Conclusions}

Fine-needle aspiration is useful for clinical management of patients with salivary glands tumors. Preoperative cytological assessment can differentiate in most cases benign versus malignant tumors, thus surgeon being able to prepare the appropriate treatment.

In some situations, due to the large variety of salivary glands entities cytological features cannot provide sufficient evidence for correct tumor characterization, the final diagnosis being established after histological studies.

\section{Acknowledgment}

The project was partly funded by the University of Medicine and Pharmacy of Tîrgu Mureș within Scientific research grants - Research groups (contract no. 20/2014).

\section{References}

1. Barnes L, Eveson JW, Reichert P, Sidransky D (Eds). World Health Organization Classification of Tumours: Pathology and Genetics of Head and Neck Tumours, IARC Press; Lyon, France 2005:212-215.

2. Trandafir V, Trandafir D, Popescu E. Tumorile maligne ale glandelor 
salivare, Jurnalul de Chirurgie, lași, 2010;6:112-131.

3. Namboodiripad APC. A review: Immunological markers for malignant salivary gland tumors, J Oral Biol Craniofac Res. 2014;4:127-134.

4. Nagao $T$, Sato $E$, Inoue $R$, et al. Immunohistochemical Analysis of Salivary Gland Tumors: Application for Surgical Pathology Practice, Acta Histochem Cytochem. 2012;45:269-282.

5. Ali NS, Akhtar S, Junaid M, et al. Diagnostic accuracy of fine needle aspiration cytology in parotid lesions. ISRN Surg. 2011;2011:721525.

6. Ansari NA, Derias NW. Origins of fine needle aspiration cytology, J Clin Pathol 1997;50;541-543.

7. Kocjan G.. Introduction and Historical Perspective, in Fine Needle Aspiration Cytology - Diagnostic Principles and Dilemmas, Springer Berlin Heidelberg, 2006:1-5.

8. Zapanta PE, Meyers AD. Fine-Needle Aspiration of the Salivary Glands, Updated: Nov. 22, 2013, http://emedicine.medscape.com/ article/882291-overview.

9. Brennan PA, Davies B, Poller D, Mead Z, Bayne D, Puxeddu R, et al. Fine needle aspiration cytology (FNAC) of salivary gland tumours: Repeat aspiration provides further information in cases with an unclear initial cytological diagnosis. Br J Oral Maxillofac Surg. 2010;48:26-29.

10. Ashraf A, Shaikh AS, Kamal F, Sarfraz R, Bukhari MH. Diagnostic reliability of FNAC for salivary gland swellings: A comparative study. Diagn Cytopathol. 2010;38:499-504.

11. Jain R, Gupta R, Kudesia M, Singh S. Fine needle aspiration cytology in diagnosis of salivary gland lesions: A study with histologic comparison. Cyto Journal. 2013;10:5.

12. Singh Nanda KD, Mehta A, Nanda J. Fine-needle aspiration cytology: a reliable tool in the diagnosis of salivary gland lesions. $\mathrm{J}$ Oral Pathol Med. 2012;41:106-112.

13. Nguansangiam S, Jesdapatarakul S, Dhanarak N, Sosrisakorn K. Accuracy of fine needle aspiration cytology of salivary gland lesions: routine diagnostic experience in Bangkok, Thailand. Asian Pac $J$ Cancer Prev. 2012;13:1583-1588.

14. Mihashi $\mathrm{H}$, Kawahara A, Kage M, et al. Comparison of preoperative fineneedle aspiration cytology diagnosis and histopathological diagnosis of salivary gland tumors. Kurume Med J 2006;53:23-27.
15. Faquin WC, Powers CN. Cap. 6 Matrix-Containing Tumors: Pleomorphic Adenoma and Adenoid Cystic Carcinoma, in Salivary Gland Cytopathology, Springer, New York 2008: 81-114.

16. Stanley MW. Selected problems in fine needle aspiration of head and neck masses. Mod Pathol. 2002;15:342-350.

17. Kocjan G. Diagnostic Dilemas in FNA Practice: Cystic Lesions, in Fine Needle Aspiration Cytology - Diagnostic Principles and Dilemmas, Springer Berlin Heidelberg, 2006:59-89.

18. Pisharodi L, Guiter G, Layfield L. Cap. 15 Fine-Needle Aspiration Biopsy, in Gnepp D.: Diagnostic Surgical Pathology of the Head and Neck, Saunders-Elsevier, Philadelphia 2009: 1069-1130.

19. Lioe TF, Elliott H, Allen DC, Spence RA. The role of fine needle aspiration cytology (FNAC) in the investigation of superficial lymphadenopathy; uses and limitations of the technique. Cytopathology. 1999;10(5):291297.

20. Das DK, Francis IM, Sharma PN, et al. Hodgkin's lymphoma: diagnostic difficulties in fine-needle aspiration cytology. Diagn. Cytopathol. 2009;37(8):564-573.

21. Jaffe ES. The 2008 WHO classification of lymphomas: implications for clinical practice and translational research. Hematology Am Soc Hematol Educ Program. 2009:523-531.

22. Kolonić SO, Prasek-Kudrna K, Roso V, et al. Value of fine-needle aspiration cytology in diagnosis of Hodgkin's lymphoma and anaplastic large cell lymphoma: one centre experience. Coll Antropol. 2010;34(1):75-79

23. Marthur S, Dawar R, Verma K. Diagnosis and grading of non-Hodgkin's lymphomas on fine needle aspiration cytology. Indian J Pathol Microbiol. 2007;50(1):46-50.

24. Elkins CT, Wakely PE Jr. Cytopathology of "double-hit" non-Hodgkin lymphoma. Cancer Cytopathol. 2011;119(4):263-271.

25. Chakrabarti S, Bera M, Bhattacharya PK, Chakrabarty D, Manna AK, Pathak S, et al.: Study of salivary gland lesions with fine needle aspiration cytology and histopothology along with immunohistochemistry. J Indian Med Assoc 2010;108:833-836.

26. Kocjan G et al. BSCC Code of Practice - fine needle aspiration cytology, Cytopathology 2009;20(5):283-296. 